\title{
Phytoprotection
}

\section{Relation entre la probabilité de survie et le choix des hôtes par les femelles : cas du parasitoïde solitaire Pachycrepoideus dubius [Hymenoptera : Pteromalidae] Relationship between offspring survival and host selection: case of a solitary parasitoid Pachycrepoideus dubius [Hymenoptera: Pteromalidae]}

\section{Marlène Goubault, Manuel Plantegenest, Liliane Krespi, Denis Poinsot, Jean-Pierre Nénon et Anne Marie Cortesero}

Volume 84, numéro 2, août 2003

$\mathrm{V}^{\mathrm{e}}$ Conférence internationale francophone d'entomologie. « La recherche de pointe en entomologie ». Montréal (Québec), Canada, 14-18 juillet 2002

URI : https://id.erudit.org/iderudit/007810ar

DOI : https://doi.org/10.7202/007810ar

\section{Aller au sommaire du numéro}

Éditeur(s)

Société de protection des plantes du Québec (SPPQ)

ISSN

0031-9511 (imprimé)

1710-1603 (numérique)

Découvrir la revue

Citer cet article

Goubault, M., Plantegenest, M., Krespi, L., Poinsot, D., Nénon, J.-P. \& Cortesero, A. (2003). Relation entre la probabilité de survie et le choix des hôtes par les femelles : cas du parasitoïde solitaire Pachycrepoideus dubius [Hymenoptera : Pteromalidae]. Phytoprotection, 84(2), 77-84. https://doi.org/10.7202/007810ar

\section{Résumé de l'article}

Chez les parasitoïdes solitaires, un seul individu émerge par hôte, aussi les femelles devraient rejeter ceux préalablement parasités. Cependant, lorsque les hôtes sains sont rares, le superparasitisme devient avantageux et les femelles devraient choisir ceux offrant les meilleures chances de survie à leurs descendants ce qui dépend du stade de développement du premier parasitoïde. Chez les ectoparasitoïdes, le premier stade larvaire est généralement considéré comme le stade combatif, seul impliqué dans la compétition larvaire. Nous avons cherché à valider ceci chez un ectoparasitoïde solitaire Pachycrepoideus dubius. Nos résultats montrent que tous les stades larvaires sont combatifs, les stades les plus âgés étant les plus aptes à remporter la compétition. Cependant, dès que le premier parasitoïde est au stade pré-nymphe, la compétition cesse et les deux individus peuvent se développer. Par ailleurs, des expériences de choix montrent que le taux d'acceptation d'hôtes hébergeant des larves de différents âges est parfaitement corrélé aux chances de survie des descendants lorsque le premier parasitoïde a moins de 10 jours. Lorsque celui-ci a atteint le stade pré-nymphe, les femelles ont tendance à rejeter ces hôtes alors que les chances de survie de leur progéniture sont d'environ $40 \%$. Cette discordance entre le choix des femelles et les chances de survie de leurs descendants est discutée. 


\title{
Relation entre la probabilité de survie et le choix des hôtes par les femelles: cas du parasitoïde solitaire Pachycrepoideus dubius [Hymenoptera : Pteromalidae]
}

\author{
Marlène Goubault ${ }^{1}$, Manuel Plantegenest ${ }^{2}$, Liliane Krespi ${ }^{1}$, \\ Denis Poinsot ${ }^{1}$, Jean-Pierre Nénon ${ }^{1}$ et Anne Marie Cortesero ${ }^{1}$
}

Reçu 2002-08-15; accepté 2003-02-21

PHYTOPROTECTION 84 : 77-84

Chez les parasitoïdes solitaires, un seul individu émerge par hôte, aussi les femelles devraient rejeter ceux préalablement parasités. Cependant, lorsque les hôtes sains sont rares, le superparasitisme devient avantageux et les femelles devraient choisir ceux offrant les meilleures chances de survie à leurs descendants ce qui dépend du stade de développement du premier parasitoïde. Chez les ectoparasitoïdes, le premier stade larvaire est généralement considéré comme le stade combatif, seul impliqué dans la compétition larvaire. Nous avons cherché à valider ceci chez un ectoparasitoïde solitaire Pachycrepoideus dubius. Nos résultats montrent que tous les stades larvaires sont combatifs, les stades les plus âgés étant les plus aptes à remporter la compétition. Cependant, dès que le premier parasitoïde est au stade pré-nymphe, la compétition cesse et les deux individus peuvent se développer. Par ailleurs, des expériences de choix montrent que le taux d'acceptation d'hôtes hébergeant des larves de différents âges est parfaitement corrélé aux chances de survie des descendants lorsque le premier parasitoïde a moins de 10 jours. Lorsque celui-ci a atteint le stade prénymphe, les femelles ont tendance à rejeter ces hôtes alors que les chances de survie de leur progéniture sont d'environ $40 \%$. Cette discordance entre le choix des femelles et les chances de survie de leurs descendants est discutée.

[Relationship between offspring survival and host selection: case of a solitary parasitoid Pachycrepoideus dubius [Hymenoptera: Pteromalidae]]

In solitary parasitoids, only one individual can emerge per host. Therefore, females should reject already parasitized hosts. However, when unparasitized hosts are scarce, superparasitism becomes advantageous and females should choose hosts that offer the best survival chances to their pro-

1. Laboratoire d'Écobiologie des insectes parasitoïdes, UPRES EA 3193, Université de Rennes 1, Campus de Beaulieu, 263, avenue du Général Leclerc, 35042 Rennes Cedex, France. Pour correspondance : marlene.goubault@univ-rennes1.fr

2. Laboratoire d'Écologie et sciences phytosanitaires, UMR ENSAR-INRA Bi03P, 65, rue de St-Brieuc - CS 84215, 35042 Rennes Cedex, France 
geny, which depend on the developmental stage of the first parasitoid. In ectoparasitoids, the first larval instar is generally considered as the fighting stage, the only one involved in larval competition. We tested this hypothesis in Pachycrepoideus dubius, a solitary ectoparasitoid. Our results show that all the larval instars are combative, the later instars winning the competition more often. Nevertheless, as soon as the first parasitoid has reached the pre-pupal stage, the competition ends and both individuals can complete their development. Moreover, choice experiments show that the acceptation rate of hosts presenting larvae of different ages is perfectly correlated to the offspring survival rate when the first parasitoid is less than 10 days old. When the first parasitoid has reached the pre-pupal stage, females tend to reject these hosts while offspring survival rate is about $40 \%$. This discrepancy between female choice and survival rate of their offspring is discussed.

\section{INTRODUCTION}

La théorie de l'approvisionnement optimal prédit que les femelles de parasitoïdes devraient choisir les hôtes les plus profitables (Charnov et Skinner 1985). La profitabilité des hôtes dépend essentiellement de leur qualité c'est-àdire, principalement, de leur espèce, leur stade de développement, leur taille et leur statut, sain ou parasité (van Alphen et Vet 1986). Le statut parasitaire des hôtes apparaît comme un facteur clé de leur qualité. En effet, pondre dans un hôte déjà parasité (i.e. superparasitisme) est souvent très coûteux (van Lenteren 1981) puisque cela réduit généralement les chances de survie des descendants des parasitoïdes solitaires. Ainsi, pour maximiser leur fitness, les femelles devraient éviter de pondre dans les hôtes déjà parasités. Néanmoins, le superparasitisme peut s'avérer adaptatif dans certaines situations et en particulier lorsque les hôtes sains sont rares (van Alphen et Visser 1990). Dans ce cas, les femelles devraient choisir, parmi les hôtes parasités, ceux qui offrent à leurs descendants la meilleure probabilité de survie. Cette probabilité dépend en particulier de l'âge et du stade de développement de l'individu déjà présent (Islam et Copland 2000; Mackauer 1990; Tillman et Powell 1992).

Chez les parasitoïdes solitaires, un seul individu peut émerger par hôte, les individus surnuméraires étant éliminés au cours de leur développement (van Lenteren 1981) par suppression physio- logique ou par combat physique (Godfray 1994; Salt 1961). Dans ce dernier cas, I'élimination résulte d'affrontements qui ont généralement lieu entre larves de même stade. Cependant, chez certains endoparasitoïdes, les larves de premier stade possédant de puissantes mandibules peuvent éliminer et parfois même consommer, des larves plus âgées, dépourvues de mandibules (Chow et Mackauer 1986; Tillman et Powell 1992). Ceci a également été observé chez certains ectoparasitoïdes, bien que chez ces espèces, tous les stades larvaires possèdent des mandibules (Godfray 1994). En effet, les larves de premier stade sont généralement les plus agressives car, pour s'installer avec succès sur un hôte, elles commencent par chercher activement les compétiteurs potentiels et les éliminent (Wai et Fujii 1990; Wylie 1971).

Pachycrepoideus dubius Ashmead [Hymenoptera: Pteromalidae] est un ectoparasitoïde s'attaquant aux pupes de diptères cyclorrhaphes (Crandell 1939). Bien qu'il s'agisse d'un parasitoïde solitaire, le superparasitisme est communément observé (Crandell 1939; Nostvik 1954). Les larves de premier stade sont très mobiles; de ce fait, elles ont été décrites comme étant impliquées dans l'élimination des individus surnuméraires (Crandell 1939). Elles pourraient éventuellement être capables d'éliminer des larves de stade plus avancé. Les femelles de $P$. dubius devraient dès lors rejeter préférentiellement les hôtes sur lesquels une larve 
de $1^{\text {er }}$ stade est présente, puisqu'un $2^{\mathrm{e}}$ individu pondu en présence d'un tel compétiteur devrait avoir une probabilité de survie très réduite.

L'influence du taux de survie probable de leurs descendants sur le choix des hôtes par les femelles a été étudié grâce à deux expériences réalisées simultanément: I'une pour évaluer les chances de survie d'un $2^{\mathrm{e}}$ individu pondu en fonction du temps écoulé depuis une $1^{\text {re }}$ ponte; et l'autre pour étudier l'effet de l'âge du $1^{\text {er }}$ parasitoïde présent sur le choix des hôtes par les femelles.

\section{MATÉRIEL ET MÉTHODES}

\section{Élevages}

L'hôte, Delia radicum L. [Diptera : Anthomyiidae], a été collecté en 1994 (Le Rheu, France). L'élevage se fait sur des racines de rutabaga (Brassica napus $L$.) dans une salle climatisée $\left(20 \pm 2{ }^{\circ} \mathrm{C} ; 60\right.$ $\pm 10 \%$ H.R.; 16L:8O) selon le protocole décrit par Neveu et al. (1996).

Deux souches de $P$. dubius, dont la couleur des yeux diffère (noir ou rouge), sont utilisées. La souche aux yeux noirs (appelée YN) a été collectée en 2000 (Rennes, France). Une mutation récessive au niveau de la couleur des yeux est apparue dans cette souche YN. Les individus mutants, qui ont les yeux rouges (appelés YR), ont été isolés et élevés séparément. Les deux souches sont élevées sur $D$. radicum dans une salle climatisée $\left(25 \pm 2{ }^{\circ} \mathrm{C} ; 60 \pm 10 \%\right.$ H.R.; 16L:8O) selon le protocole décrit par Grandgirard et al. (2002).

\section{Protocoles expérimentaux}

\section{Taux de survie}

Vingt femelles vierges de $P$. dubius ont été placées ensemble en présence de 20 pupes saines de $D$. radicum pendant $4 \mathrm{~h}$. Les femelles $\mathrm{YN}$ et YR ont pondu séparément. Ces pontes ont eu lieu tous les j à la même h. Dans les 28 h qui suivaient le début des pontes (i.e. avant l'éclosion), les pupes ont été disséquées afin de récolter les œufs. Une fente a ensuite été réalisée dans le puparium de pupes saines à l'aide d'une aiguille. Les œufs récoltés de $P$. dubius ont alors été introduits par cette fente et déposés sur les pupes de $D$. radicum. Les pupes utilisées étaient âgées de 4 à $6 \mathrm{j}$ car des tests préliminaires avaient montré que, sur de tels hôtes, le taux de survie des individus manipulés était similaire au taux observé lors d'un parasitisme naturel (soit environ $60 \%$ de chances de survie). Ici, deux œufs ont été déposés par hôte, un de chaque souche à différents intervalles de temps : $0 \mathrm{j}(0-4 \mathrm{~h})$, $1 \mathrm{j}, 2 \mathrm{j}, 3 \mathrm{j}, 5 \mathrm{j}, 7 \mathrm{j}, 10 \mathrm{j}, 15 \mathrm{j}$ et $18 \mathrm{j}$. Á ces intervalles de temps, le $1^{\text {er }}$ parasitoïde devait être respectivement un œuf jeune, un œuf âgé, une larve de $1^{\text {er }}$ stade (L1), de $2^{\mathrm{e}}$ stade (L2), de $3^{\mathrm{e}}$ stade (L3), de $5^{\mathrm{e}}$ stade (L5), une pré-nymphe, une nymphe et un pré-adulte. Les deux ordres de ponte ont été testés, i.e. YN puis $Y R$ et YR puis YN (Tableau 1). Un

Tableau 1. Détail des effectifs par traitement : nombre de pupes de Delia radicum dans lesquelles ont été placés deux œufs de deux souches différentes (YN ou YR) de Pachycrepoideus dubius en fonction de l'intervalle de temps séparant le dépôt artificiel de ces deux œufs et de l'ordre de leur dépôt

\begin{tabular}{lcccccccccc}
\hline & \multicolumn{8}{c}{ Intervalle de temps entre les deux pontes (jours) } \\
\cline { 2 - 10 } & $\mathbf{0}$ & $\mathbf{1}$ & $\mathbf{2}$ & $\mathbf{3}$ & $\mathbf{5}$ & $\mathbf{7}$ & $\mathbf{1 0}$ & $\mathbf{1 5}$ & $\mathbf{1 8}$ \\
\cline { 2 - 10 } Traitement & $\begin{array}{c}\text { Fuf } \\
\text { jeune }\end{array}$ & Fuf âgé & L1 & L2 & L3 & L5 & $\begin{array}{c}\text { Pré- } \\
\text { nymphe }\end{array}$ & Nymphe & $\begin{array}{c}\text { Pré- } \\
\text { adulte }\end{array}$ \\
\hline YN puis YR & 160 & 51 & 55 & 54 & 50 & 50 & 50 & 49 & 49 \\
YR puis YN & 52 & 57 & 58 & 50 & 47 & 48 & 49 & 48 \\
\hline
\end{tabular}


témoin sans compétition a été obtenu en déposant un seul œuf par pupe saine (YN : $N=49 ; Y R: N=48$ ). L'influence du temps écoulé depuis la première ponte sur les chances de survie des descendants a d'abord été testée séparément pour les deux ordres de ponte, puis elle a été testée en regroupant ces données.

\section{Sélection des hôtes}

Les femelles $Y N$ testées ( $N=24)$, accouplées et expérimentées, ont été placées individuellement avec 10 hôtes de cinq types différents $\left(22 \pm 1{ }^{\circ} \mathrm{C} ; 40 \pm 10 \%\right.$ H.R.) : 2 étaient parasités depuis $0 \mathrm{j}$ (0-4 h), 2 depuis $1 \mathrm{j}, 2$ depuis $2 \mathrm{j}, 2$ depuis $5 \mathrm{j}$ et 2 depuis $10 \mathrm{j}$. Ces hôtes avaient été parasités (de manière naturelle) par des femelles conspécifiques. La position relative de ces hôtes a été tirée au sort et notée sur un schéma afin d'identifier les hôtes. Les acceptations et rejets de ces hôtes ont été observés pendant $2 \mathrm{~h}$. Pour éviter de confondre l'effet de la discrimination polygyne (reconnaissance des hôtes exploités par des congénères) et celui de la discrimination monogyne (reconnaissance des hôtes que la femelle testée a elle-même exploités au cours de l'expérience), seule la première rencontre avec chaque pupe a été prise en compte. Les résultats ont alors été regroupés par type d'hôtes.

\section{Analyses statistiques}

L'effet du temps écoulé entre les pontes a été analysé avec le modèle linéaire généralisé (GLIM), sous I'hypothèse d'un lien logit et d'une distribution binomiale (Mc Cullagh et Nelder 1989). Les analyses ont été réalisées avec la procédure GENMOD du logiciel SAS (Version 3.04, SAS Institute inc. 1996). Les comparaisons des fréquences ont été réalisées avec des tests du chi carré. La comparaison des taux d'acceptation des hôtes en fonction du temps écoulé depuis le premier parasitisme de ces hôtes a été faite grâce à une régression logistique effectuée sur les taux d'acceptation après transformation arcsinus (racine). Cette transformation a également été appliquée lors de la recherche de corrélation entre les taux d'acceptation des hôtes et des chances de survie des descendants sur ces hôtes.

\section{RÉSULTATS}

\section{Taux de survie}

Le taux d'émergence en l'absence de compétiteur ne diffère pas significativement entre les deux souches $\left(\chi^{2}{ }_{(1)}=1,50\right.$; $P=0,22$ ) et atteint une moyenne $67 \%$ (Tableau 2). En situation de compétition, la proportion de pupes desquelles a émergé au moins un individu est indépendante de l'intervalle de temps entre les pontes (GLIM, $\chi^{2}{ }_{(17)}=16,50 ; P$ $=0,49$ ) (Tableau 2).

Les taux de survie du $1^{\text {er }}$ comme du $2^{\mathrm{e}}$ individu dépendent du temps écoulé entre les pontes $\left(\chi^{2}{ }_{(7)}=41,11 ; P<0,001\right.$ et $\chi_{(7)}^{2}=98,71 ; P<0,001$, respectivement) (Tableau 3 ) mais pas de la souche $\left(\chi^{2}{ }_{(1)}=2,25 ; P=0,13\right.$ et $\chi_{(1)}^{2}=3,00 ; P=$ 0,08 , respectivement) (Tableau 3 ). Toutefois, lorsque les deux œufs sont pondus simultanément $(0 \mathrm{j}$ soit $0-4 \mathrm{~h}$ d'intervalle), les individus de la souche YN émergent significativement plus souvent que ceux de la souche YR (77

Tableau 2. Proportion (\%) de pupes de Delia radicum desquelles émerge au moins un individu du parasitoïde Pachycrepoideus dubius en fonction de l'intervalle de temps entre le dépôt artificiel de deux œufs de deux souches différentes (YN ou YR) sur ces pupes et de I'ordre du dépôt de ces œufs

\begin{tabular}{|c|c|c|c|c|c|c|c|c|c|c|}
\hline \multirow{2}{*}{ Traitement } & \multirow{2}{*}{$\begin{array}{l}1 \text { seule } \\
\text { ponte }\end{array}$} & \multicolumn{9}{|c|}{ Intervalle de temps entre les deux pontes (jours) } \\
\hline & & 0 & 1 & 2 & 3 & 5 & 7 & 10 & 15 & 18 \\
\hline YN puis YR & 0,61 & \multirow{2}{*}{0,80} & 0,73 & 0,67 & 0,63 & 0,66 & 0,68 & 0,72 & 0,73 & 0,62 \\
\hline YR puis YN & 0,73 & & 0,75 & 0,65 & 0,67 & 0,76 & 0,68 & 0,63 & 0,71 & 0,85 \\
\hline
\end{tabular}


Tableau 3. Taux de survie (\%) d'un $1^{\text {er }}$ et $d^{\prime}$ un $2^{\mathrm{e}}$ individu du parasitoïde Pachycrepoideus dubius en fonction de l'intervalle de temps entre le dépôt artificiel de deux œufs de deux souches différentes (YN ou YR) sur une pupe de Delia radicum et de l'ordre du dépôt de ces œufs

\begin{tabular}{lccccccccc}
\hline & \multicolumn{1}{c}{ Intervalle de temps entre les deux pontes (jours) } \\
\cline { 2 - 10 } Traitement & $\mathbf{0}$ & $\mathbf{1}$ & $\mathbf{2}$ & $\mathbf{3}$ & $\mathbf{5}$ & $\mathbf{7}$ & $\mathbf{1 0}$ & $\mathbf{1 5}$ & $\mathbf{1 8}$ \\
\hline $\mathbf{1}^{\text {er }}$ individu \\
$\begin{array}{l}\text { YN puis YR } \\
\text { YR puis YN }\end{array}$ & 0,50 & 0,37 & 0,38 & 0,42 & 0,54 & 0,66 & 0,64 & 0,71 & 0,55 \\
$\mathbf{2}^{\mathrm{e}}$ individu & 0,30 & 0,54 & 0,47 & 0,41 & 0,72 & 0,68 & 0,40 & 0,65 & 0,81 \\
YN puis YR & & & & & & & & & \\
YR puis YN & 0,50 & 0,22 & 0,17 & 0,26 & 0,04 & 0,00 & 0,35 & 0,35 & 0,44 \\
& 0,30 & 0,35 & 0,29 & 0,20 & 0,12 & 0,02 & 0,44 & 0,37 & 0,43 \\
\hline
\end{tabular}

émergents $\mathrm{YN}$ vs 47 émergents $\mathrm{YR} ; \chi^{2}{ }_{(1)}$ $=11,85 ; P<0,001)$. Cependant, cet avantage de la souche $\mathrm{YN}$ disparaît dès que le temps écoulé entre les pontes est d'au moins $1 \mathrm{j}$. Ensuite, le taux de survie du $1^{\text {er }}$ individu augmente progressivement, lorsque l'intervalle de temps entre les pontes s'accroît, jusqu'à atteindre environ $70 \%$, taux non significativement différent de celui observé en l'absence de compétiteur $\left(\chi_{(1)}^{2}=0,56\right.$; $P=0,45$ ) (Fig. 1). Le taux de survie du $2^{\mathrm{e}}$ individu diminue progressivement jusqu'à être nul lorsque le $1^{\text {er }}$ individu a atteint le $5^{\mathrm{e}}$ stade larvaire (7 j) (Fig. 1).
Puis, son taux de survie augmente sans affecter celui du $1^{\text {er }}$ individu. En effet, à ce stade, les deux individus peuvent achever leur développement et émerger du même hôte.

Ainsi, alors qu'aucune émergence double n'est observée lorsque l'intervalle de temps entre les pontes est inférieur à $10 \mathrm{j}$, à partir de $10 \mathrm{j}$ d'écart entre les deux pontes, $44 \%$ des individus émergés proviennent d'émergences doubles. Ces émergences doubles représentent $79 \%$ des émergences pour le $2^{\mathrm{e}}$ individu.

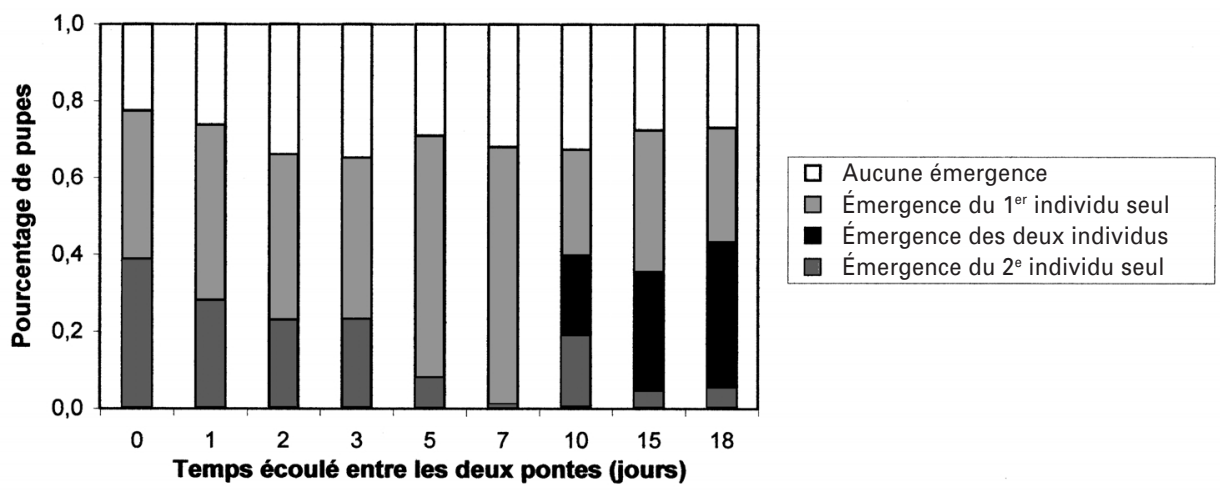

Figure 1. Proportion relative des issues possibles du parasitisme des pupes de Delia radicum par le parasitoïde Pachycrepoideus dubius, en fonction du temps écoulé entre le dépôt artificiel de deux œufs de ce parasitoïde. 


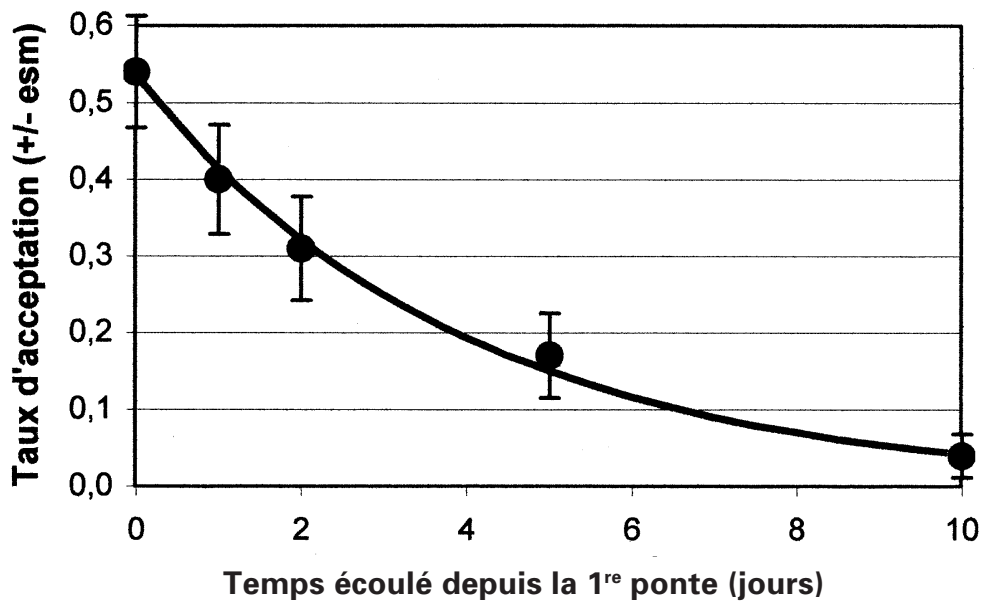

Figure 2. Taux d'acceptation des pupes de Delia radicum déjà parasitées par les femelles de Pachycrepoideus dubius, en fonction du temps écoulé depuis la première ponte de ce parasitoïde (après transformation : -Ln (arcsinus (racine (taux d'acceptation)) = 0,22 + 0,14 (intervalle de temps entre les pontes); $R=0,99 ; F=949,00 ;$ d.I. $=4 ; P<0,0001$ ).

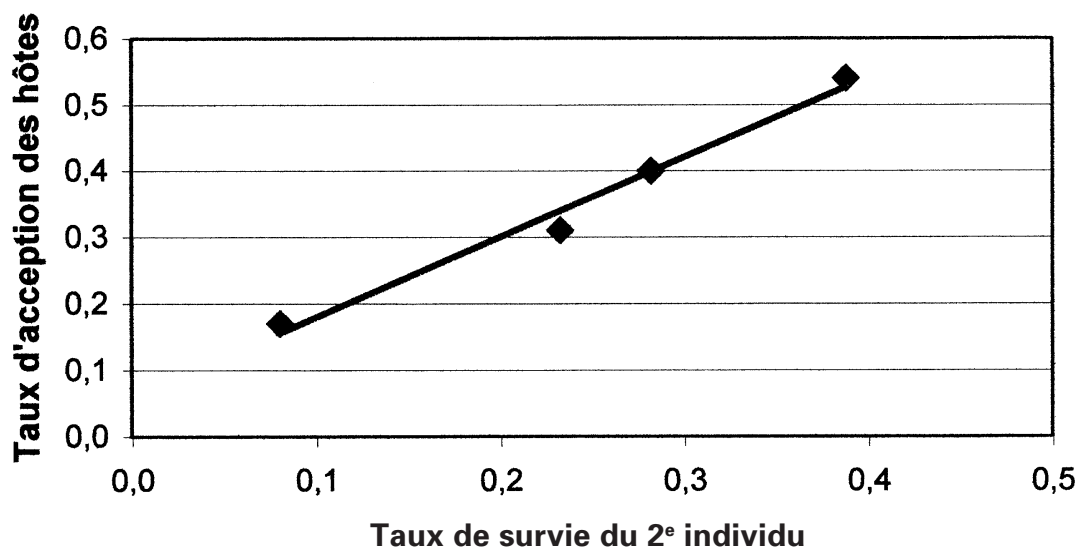

Figure 3. Corrélation entre le taux d'acceptation des pupes de Delia radicum (déjà parasitées depuis moins de 10 jours) par les femelles de Pachycrepoideus dubius et le taux de survie des $2^{\text {es }}$ individus pondus par ces femelles sur ces hôtes.

\section{Sélection des hôtes}

La fréquence d'acceptation des hôtes décroît avec le temps écoulé depuis la première ponte après transformation logarithmique $(R=0,99 ; F=949,00 ;$ d.I. $=4 ; P<0,0001)$ (Fig. 2).

\section{Relation entre la probabilité de survie des descendants et la sélection des hôtes}

Le taux d'acceptation par les femelles des hôtes parasités depuis moins de 10 j est linéairement corrélé à la probabilité de survie des descendants dans ces hôtes $(R=0,99 ;$ d.I. $=3 ; P<0,01)$ (Fig. 3). Pour les hôtes parasités depuis plus de $10 \mathrm{j}$, la corrélation n'existe plus : le taux d'acceptation des hôtes est encore 
de $39,80 \pm 4,97 \%$ alors que la probabilité de survie des descendants n'est plus que de $4,17 \pm 2,88 \%$.

\section{DISCUSSION}

En situation de superparasitisme, I'âge et/ou le stade de développement du premier individu installé jouent un rôle essentiel dans le succès reproducteur des parasitoïdes solitaires (Islam et Copland 2000; Mackauer 1990; Tillman et Powell 1992). L'effet du temps écoulé entre deux pontes sur la survie a été évalué en utilisant deux souches, $\mathrm{YN}$ et YR, de P. dubius. La compétition apparaît symétrique, excepté dans le cas d'un dépôt simultané des œufs qui conduit le plus souvent à l'émergence de l'individu YN. Cette asymétrie pourrait être due à une éclosion légèrement plus précoce des œufs de la souche YN.

Lorsqu'il y a un décalage temporel entre les pontes, la probabilité de survie du $2^{\mathrm{e}}$ individu $\mathrm{s}^{\prime}$ amenuise lorsque le délai entre les pontes s'accroît, jusqu'à s'annuler lorsque le $1^{\text {er }}$ individu atteint le $5^{\mathrm{e}}$ stade larvaire $(7 \mathrm{j})$. Ainsi, chez $P$. dubius, l'aptitude à la compétition existe chez tous les stades larvaires et s'accroît avec l'âge, contrairement à ce qui a été avancé par Crandell (1939) et observé chez d'autres ectoparasitoïdes (Wai et Fujii 1990; Wylie 1971).

Lorsque le $1^{\text {er }}$ individu a passé le $5^{\mathrm{e}}$ stade, la probabilité de survie du $2^{\mathrm{e}}$ individu augmente sans affecter celle du $1^{\text {er }}$ individu. En fait, lorsque le $1^{\text {er }}$ individu a atteint ou passé le stade prénymphe (10 j), la compétition cesse et les deux individus peuvent achever leur développement. Les observations d'émergences doubles à partir d'un même hôte indiquent que $P$. dubius n'est pas un solitaire strict sur des pupes de grande taille (telles que celles de $D$. radicum). Elles suggèrent, $d^{\prime}$ autre part, que les individus éliminés ne le sont ni par manque de nourriture ni par asphyxie mais par action directe des compétiteurs. Des observations complémentaires ont montré que les larves s'éliminaient au cours de combats pouvant conduire au cannibalisme des individus tués (Goubault, non publié).
Concernant la sélection des hôtes, les femelles de $P$. dubius montrent une préférence pour les hôtes récemment parasités et un taux d'acceptation des hôtes parasités depuis moins de $10 \mathrm{j}$ parfaitement corrélé aux chances de survie des descendants. Elles seraient donc capables d'estimer la variation de la qualité de I'hôte due à l'âge du $1^{\text {er }}$ parasitoïde, comme cela a déjà été observé chez d'autres espèces (Tillman et Powell 1992). Ainsi, les femelles de $P$. dubius semblent choisir les hôtes les plus profitables, comme le prédit la théorie de l'approvisionnement optimal (Charnov et Skinner 1985). Toutefois, les hôtes parasités depuis $10 \mathrm{j}$ sont quasiment toujours rejetés alors qu'ils offrent de bonnes chances de survie aux descendants (possibilités d'émergences doubles). Ceci pourrait être lié à la réduction de taille des $2^{\text {es }}$ individus issus d'émergences doubles (Goubault, observations personnelles), paramètre fréquemment corrélé avec la fitness individuelle (Voir cependant Roitberg et al. 2001). Ainsi, les femelles éviteraient de parasiter ces hôtes qui, bien qu'offrant de bonnes chances de survie à leur progéniture, ne permettraient le développement que de petits individus, à faible fitness.

\section{REMERCIEMENTS}

Nous remercions S. Dourlot, M. Rault et C. Jammoneau-Paty pour leur assistance technique et Guy Boivin et Joan van Baaren pour leurs précieux conseils. $M$. Goubault bénéficie d'une allocation du Ministère français de la Recherche. 


\section{RÉFÉRENCES}

Charnov, E.L. et S.W. Skinner. 1985. Complementary approaches to the understanding of parasitoid oviposition decisions. Environ. Ecol. 14 : 383-391.

Chow, F.J. et M. Mackauer. 1986. Host discrimination and larval competition in the aphid parasite Ephedrus californicus. Entomol. Exp. Appl. 41 : 243-254.

Crandell, H.A. 1939. The biology of Pachycrepoideus dubius Ashmead (Hymenoptera), a Pteromalid parasite of Piophila casei Linne. Entomol. Soc. Am. 32 : 632-654.

Godfray, H.C.J. 1994. Parasitoids: Behavioral and evolutionary ecology. Princeton University Press, Princeton. 473 pp.

Grandgirard J., D. Poinsot, L. Krespi, J.P. Nénon et A.M. Cortesero. 2002. Costs of secondary parasitism in the facultative hyperparasitoid, Pachycrepoideus dubius: does host size matter? Entomol. Exp. Appl.103: 239-248.

Islam, K.S. et M.J.W. Copland. 2000. Influence of egg load and oviposition time interval on the host discrimination and offspring survival of Anagrus pseudococci (Hymenoptera : Encyrtidae), a solitary endoparasitoid of citrus maelybug, Planococcus citri (Hemiptera: Pseudococcidae). Bull. Entomol. Res. 90 : 69-75.

Mackauer, M. 1990. Host discrimination and larval competition in solitary endoparasitoids. Pages 41-62 in M. Mackauer, L.E. Ehler et J. Roland (éds.), Critical issues in biological control. Intercept, Andover, U.K.

Mc Cullagh, P. et J.A. Nelder. 1989. Generalized linear models, $2^{\mathrm{e}}$ éd. Chapman \& Hall, London. 511 pp.

Neveu, N., N. Kacem et J.P. Nénon. 1996. A method for rearing Trybliographa rapae W. on Delia radicum L. OILB / SROP Bulletin $19: 173-178$.
Nostvik, E. 1954. Biological studies of Pachycrepoideus dubius Ashmead (Chalcidoidae : Pteromalidae), a pupal parasite of various Diptera. Oikos 5 : 195-204.

Roitberg, B.D., G. Boivin et L.E.M. Vet. 2001. Fitness, parasitoids, and biological control: an opinion. Can. Entomol. 133 : 429438.

Salt, G. 1961. Competition among insect parasitoids. Symp. Soc. Exp. Biol. 15 : 96119.

Tillman, P.G. et J.E. Powell. 1992. Intraspecific host discrimination and larval competition in Microplitis croceipes, Microplitis demolitor, Cotesia kazak (Hym. : Braconidae) and Hyposoter didymator (Hym. : Ichneumonidae), parasitoids of Heliothis virescens (Lep. : Noctuidae). Entomophaga 37 : 229237.

van Alphen, J.J.M. et L.E.M. Vet. 1986. An evolutionary approach to host finding. Pages 23-62 in J. Waage et D. Greathead (éds.), Insects parasitoid. Academic Press, New York.

van Alphen, J.J.M. et M.E. Visser. 1990. Superparasitism as an adaptive strategy for insect parasitoids. Annu. Rev. Entomol. 35 : 59-79.

van Lenteren, J.C. 1981. Host discrimination by parasitoids. Pages 163-179 in D.A. Nordlund, R.L. Jones et W.J. Lewis (éds.), Semiochemicals, their role in pest control. John Wiley, New York.

Wai, K.M. et K. Fujii. 1990. Intraspecific larval competition among wasps parasitic of bean weevil larvae. Res. Popul. Ecol. 32 : 85-98.

Wylie, H.G. 1971. Observations on intraspecific larval competition in three $\mathrm{Hy}$ menopterous parasites of fly puparia. Can. Entomol. 103 : 137-142. 